Gilbert Blaise $\mathrm{MD}$, Eric Troncy DVM MSc

\title{
The best approach to ARDS?
}

modulate the inflammatory response and modify the disease process causing the ARDS. Inhaled prostacyclin also improve oxygenation and has been successful in patients suffering from ARDS. The combination of these two treatments might have an additive effect on oxygenation as they act through different mechanisms of vasodilatation. Inhaled NO, by stimulating soluble guanylate cyclase, increases cyclic 3' -5 " guanosine monophosphate (cGMP) and prostacyclin, by stimulating adenylate cyclase, increases cAMP levels. The effects of these vasodilators may be increased by medications such as phosphodiesterases 3 and 5 blockers which diminish catabolism of CAMP and cGMP respectively. It has been shown that zaprinast (Ziprinist ${ }^{\mathrm{TM}}$ ) and pyridamol (Persantin ${ }^{\mathrm{TM}}$ ) potentiate the pulmonary effects of inhaled NO. Zaprinast was also recently tested with success in aerozilation of experimental pulmonary hypertension. ${ }^{3}$

Vasoconstrictors which will facilitate pulmonary vasoconstriction by decreasing flow to low ventilation/ perfusion ratio lung units might by themselves increase pulmonary pressure, improve oxygenation and potentiate the effect of local vasodilators such as inhaled NO and inhaled prostacyclin. Almitrine is a pulmonary vasoconstrictor which potentiates hypoxic pulmonary vasoconstriction and increases cardiac output. It has been used either alone or in combination with inhaled NO to improve oxygenation in ARDS. Both medications have an additive effect on oxygenation and opposite effect on pulmonary pressure. Is this action specific for almitrine or is it the potential of any vasoconstrictor? One publication $^{4}$ suggested that phenylephrine may be synergistic with inhaled NO in ARDS patients despite a sparse distribution of alpha receptors on pulmonary arteries. Moreover, due to limitations in the study design, these results must be approached with prudence. The association of other vasoconstrictors such as angiotensin and vasopressin and inhaled NO has not been tested.

In supine ARDS patients, the dependent dorsal pulmonary regions are collapsed under the increased weight of the oedema-laden lungs. Turning the patients suffering from severe ARDS from supine to prone 
position improves oxygenation. Proposed explanations include a prone position-induced (1) recruitment of previously collapsed alveoli; (2) increase in functional residual capacity; (3) change in regional diaphragm motion; (4) redistribution of perfusion along a gravitational gradient toward less injured lung regions associated with (5) a better drainage of secretions. The effect of positioning on ventilation could be long-lasting even after returning to supine position and potentiates the effect of pulmonary vasoactive medications on oxygenation.

In this issue of the Canadian Journal of Anaesthesia, Gillart et al. ${ }^{1}$ have demonstrated additive effects of inhaled NO, almitrine and ventilation in the prone position. They studied patients over a short time, therefore we cannot conclude if this potentiation of the three techniques in improving oxygenation is long-lasting. Their findings are consistent with results of a Swiss group ${ }^{5}$ that, by acting through different mechanisms on ventilation/perfusion relationships, the prone position, inhaled NO, and almitrine may exert additive beneficial effects on gas exchange in the lungs of ARDS patients.

Recently, new techniques of ventilation have been used to improve oxygenation or to prevent ventilationinduced pulmonary trauma. Those worth mentioning include: liquid (perfluorocarbon) ventilation, high frequency ventilation, inverse ratio ventilation and protective ventilation (low tidal volume, low inspiratory pressure) with permissive hypercapnia. Even if this last mode of mechanical ventilation, which reduces inspiratory pressure and tidal volume, is effective in reducing lung trauma in animals, its preventive beneficial effect in humans with ARDS is difficult to show. Indeed, if Amato $e t a l^{6}$ have shown an impressive advantage of protective mechanical ventilation, compared with classical mechanical ventilation, on short term mortality, weaning from mechanical ventilation and the incidence of barotrauma. Long term survival rate was not modified by protective ventilation. Others, in a large retrospective study, ${ }^{7}$ have not found a relation between ventilatory pressure and pneumothorax and Stewart et al..$^{8}$ even found an increase in morbidity in patients whose lungs were ventilated with protective pressure limit ventilation. As suggested three years ago, ${ }^{9}$ it has not been possible to demonstrate the efficacy of any new treatment on mortality in ARDS over the last 20 years, even if the incidence and the global mortality of the pathology have decreased during the same period. In future it will be very difficult or impossible to demonstrate that a single change in ventilatory treatment could have an effect on mortality in ARDS patients. The future is turned rather toward therapeutic optimisation including $(1)$ decrease in extravascular lung water (diuretics or haemofiltration); (2) selection of the best ventilatory mode (PEEP level, pressure-controlled with inverse ratio ventilation, etc.); (3) permissive hypercapnia; (4) vasoactive support (adrenergic drugs, perfusion, transfusion, etc.); (5) treatment of infection and corticosteroids; and (6) correction of hypoxaemia by additional continuous tracheal gas insufflation, inhaled NO inducing pulmonary vasodilation and steal phenomenon, almitrine (or other vasoconstrictor) increasing the hypoxic pulmonary vasoconstriction, prone position helping to the alveolar recruitment and better ventilation/perfusion matching, and drainage of pleural or mediastinal effusions. Patients responding rapidly to the optimised treatment had a better prognosis. ${ }^{10}$ We have several tools to treat the respiratory aspect of ARDS, but patients rarely die from respiratory failure. The indication to treat these patients with ECMO has almost disappeared and the new challenge is to prevent or to modulate adequately (either inhibition or stimulation) the systemic inflammatory response and prevent the multiple organ failure which kill these patients. Control of the NO pathway, either by selective inhibition of NO synthase, or by administration of exogenous $\mathrm{NO}$, is one of the means to modulate the inflammatory response.

\section{Quelle est la meilleure approche au SDRA?}

Dans ce numéro, Gillart et al. ${ }^{1}$ rapportent les effets sur l'oxygénation de trois modalités thérapeutiques utilisées de façon isolée ou ensemble chez des patients présentant un SDRA sévère et bien établi. Cette pathologie est caractérisée par des infiltrats pulmonaires bilatéraux dus à de l'oedème pulmonaire secondaire à une perméabilité élevée, à de l'atélectasie, de l'infection, de l'hypertension pulmonaire secondaire à la vasoconstriction et à l'occlusion vasculaire par des leucocytes et des agrégats plaquettaires, ainsi qu'une compliance faible et de l'hypoxémie. Cette dernière est principalement due à des inégalités ventilation-perfusion $(\mathrm{V} / \mathrm{Q})$. Le sang provenant des alvéoles mal ventilées (rapport $\mathrm{V} / \mathrm{Q}$ faible) et mal oxygénées se mélange dans les veines pulmonaires avec le sang complètement oxygéné provenant des alvéoles avec rapport $\mathrm{V} / \mathrm{Q}$ normal, causant une admission veineuse, réalisant un effet shunt. Les objectifs du traitement pour les patients souffrant de SDRA sont d'améliorer l'oxygénation, d'éviter tout trauma pulmonaire additionnel et d'aider le poumon à guérir. 
Le médecin a en mains plusieurs approches possibles pour améliorer l'oxygénation. La première est d'accroître la fraction inspirée d'oxygène $\left(\mathrm{FiO}_{2}\right)$; tous les patients sont ventilés avec un mélange gazeux contenant une haute concentration d'oxygène. La seconde et plus fréquemment utilisée est la pression positive de fin d'expiration (PEEP) qui, par son effet de recrutement des alvéoles, diminue la quantité et l'intensité des zones de bas rapport $V / Q$ dans le poumon. Plus récemment, de nouvelles médications, de nouveaux modes de ventilation et de positionnement ont démontré des effets bénéfiques sur la fonction pulmonaire immédiate. L'adéquation ventilation/perfusion peut être favorisée soit en augmentant le flot sanguin aux alvéoles bien ventilées ou en diminuant le flot aux alvéoles mal ventilées. Pour améliorer l'oxygénation, les vasodilatateurs doivent agir principalement sur les vaisseaux perfusant les alvéoles ventilées et c'est pour cette raison que les vasodilatateurs doivent être administrés par les voies aériennes pour avoir la sélectivité suffisante pour améliorer l'oxygénation. Parmi les nouvelles thérapies, on retrouve l'oxyde nitrique (NO). Son effet de vasodilatation pulmonaire sur les vaisseaux perfusant les alvéoles ventilées produit un phénomène de vol sanguin vers les alvéoles ventilées améliorant l'oxygénation et diminuant la pression pulmonaire ${ }^{2}$. Ses effets sur les plaquettes et sur l'activation leucocytaire peuvent aussi moduler la réponse inflammatoire et modifier le processus morbide causant le SDRA. Des protacyclines administrées par inhalation améliorent aussi l'oxygénation et ont été utilisées avec succès chez des patients souffrant de SDRA. La combinaison de ces deux traitements pourrait avoir un effet additif sur l'oxygénation dans la mesure où ils agissent par différents mécanismes de vasodilatation. Le NO inhalé, en stimulant la guanylate cyclase soluble, augmente la concentration de guanosine monophosphate cyclique $3^{1}-5^{1}$ (cGMP) de même que la prostacycline qui par sa stimulation de l'adénylate cyclase augmente les niveaux de cAMP. Les effets de ces vasodilatateurs peuvent être augmentés par des médications telles que les inhibiteurs des phosphodiestérases 3 et 5 qui diminuent respectivement le catabolisme de la cAMP et de la cGMP. On a démontré que le zaprinast (Ziprinist@) et que le pyridamole (Persantin $(\mathcal{O})$ potentialisent les effets pulmonaires du NO inhalé. Récemment, on a aussi testé avec succès le zaprinast en aérosolisation dans l'hypertension pulmonaire expérimentale ${ }^{3}$.

Les vasoconstricteurs qui facilitent la vasoconstriction pulmonaire en diminuant le flot aux unités pulmonaires avec $\mathrm{V} / \mathrm{Q}$ bas pourraient aussi augmenter la pression pulmonaire, améliorer l'oxygénation et potentialiser l'effet des vasodilatateurs comme le NO et la prostacycline inhalés. L'almitrine est un vasoconstricteur pulmonaire qui potentialise la vasoconstriction pulmonaire hypoxique et augmente le débit cardiaque. L'almitrine a été utilisé seul ou en combinaison avec le NO inhalé pour améliorer l'oxygénation dans le SDRA. Les deux médications ont un effet additif sur l'oxygénation et un effet opposé sur la pression pulmonaire. Cet effet est-il spécifique à l'almitrine ou est-il le même pour tous les vasoconstricteurs? Une publication ${ }^{4}$ suggère que la phényléphrine pourrait avoir un effet synergique avec le NO inhalé chez les patients présentant un SDRA malgré la faible distribution des récepteurs alpha au niveau des artères pulmonaires. Cependant, à cause des limites du protocole de l'étude, ces résultats doivent être considérés avec prudence. L'association d'autres vasoconstricteurs tels l'angiotensine et la vasopressine avec le NO inhalé n'a pas été expérimentée.

Chez les patients avec SDRA en position dorsale, les régions pulmonaires dépendantes sont collabées sous le poids accru des poumons chargés d'œdème. Le fait de tourner ces patients de la position dorsale à la position ventrale améliore l'oxygénation. Les explications proposées comportent les éléments suivants provoqués par le décubitus ventral : 1-un recrutement d'alvéoles déjà collabées 2 -une augmentation de la capacité résiduelle fonctionnelle 3-une modification régionale des mouvements diaphragmatiques 4-une redistribution de la perfusion selon un gradient lié à la gravité vers les régions pulmonaires moins atteintes 5-un meilleur drainage des sécrétions. L'effet sur la ventilation du changement de position peut être durable et persister même après le retour en décubitus dorsal et potentialiser les effets des médicaments vasoactifs pulmonaires sur l'oxygénation.

Dans ce numéro, Gillart $e t a l{ }^{1}$ ont montré les effets additifs du NO inhalé, de l'almitrine et de la ventilation en décubitus ventral. Ils ont érudié les parients durant une courte période de temps, ce qui ne nous permet pas de conclure que l'amélioration de l'oxygénation par ces trois techniques soit de longue durée. Leurs trouvailles concordent avec les résultats d'un groupe suisse ${ }^{5}$ à savoir que, bien qu'agissant par des mécanismes différents sur le rapport $\mathrm{V} / \mathrm{Q}$, le décubitus ventral, le NO inhalé et l'almitrine peuvent avoir des effets bénéfiques additifs sur les échanges gazeux dans les poumons de patients atteints de SDRA.

Récemment, on a utilisé de nouvelles techniques de ventilation pour améliorer l'oxygénation ou pour prévenir le barotrauma pulmonaire due à cette ventilation. Celles qui valent la peine d'être mentionnées sont : la ventilation liquide (avec perfluorocarbone), la ventilation à haute fréquence, la ventilation à rapports 
inversés et la ventilation protectrice (volume courant et pression inspiratoire faibles) avec hypercarbie permissive. Même si ce dernier mode de ventilation mécanique, qui utilise des volumes et pression réduites, réduit de façon efficace les trauma pulmonaires chez les animaux, son effet bénéfique préventif est difficile à démontrer chez les humains avec SDRA. En effet, bien que Amato et al. ${ }^{6}$ aient montré un avantage marqué de la ventilation mécanique protectrice, par rapport à la ventilation conventionnelle, sur la mortalité à court terme, sur le sevrage de la ventilation mécanique et sur l'incidence de barotrauma, la survie à long terme n'a pas été modifiée. D'autres, dans une étude rétrospective étendue $^{7}$, n'ont pas trouvé de relation entre la pression de ventilation et le pneumothorax et Stewart $e t a l .^{8}$ ont même trouvé une augmentation de morbidité chez les patients ventilés avec une ventilation avec limite de pression protectrice. Tel que suggéré il y a $\mathbf{3}$ ans, ${ }^{9}$ il n'a pas été possible de démontrer l'efficacité d'aucun nouveau traitement sur la mortalité en SDRA depuis 20 ans, même si l'incidence et la mortalité globale de cette pathologie ont diminué durant la même période. Dans le futur, il sera très difficile voire impossible de démontrer qu'un changement isolé dans le traitement ventilatoire puisse avoir un effet sur la mortalité dans le SDRA. Le futur réside plutôt dans une optimisation de la thérapeutique, ce qui inclura : 1-une diminution de l'eau pulmonaire extravasculaire (diurétiques ou hémofiltration), 2-choix du meilleur mode ventilatoire (niveau de PEEP, contrôle de pression, rapports inversés, etc.), 3-hypercarbie permissive, 4-support vasoactif (médicaments adrénergiques, perfusions, transfusions, etc.), 5-traitement de l'infection et corticostéroïdes, 6correction de l'hypoxémie par insufflation additionnelle continue de gaz dans la trachée, inhalation de NO pour produire de la vasodilatation pulmonaire et des phénomènes de vol, de l'almitrine (ou d'autres vasoconstricteurs) pour accroître la vasoconstriction pulmonaire hypoxique, le décubitus ventral pour favoriser le recrutement alvéolaire et l'adéquation de la ventilation/perfusion, ainsi que le drainage des épanchements pleuraux ou mé-diastinaux. Les patients qui répondent rapidement au traitement ont un meilleur pronostic ${ }^{10}$. Nous avons plusieurs armes pour traiter l'aspect respiratoire du SRDA mais les patients meurent rarement d'insuffisance respiratoire. L'indication de traiter ces patients avec ECMO (oxygénation à membrane extra-corporelle) est presque disparue et le nouveau défi consiste à prévenir ou à moduler adéquatement (soit par stimulation soit par inhibition) la réponse inflammatoire systémique et à prévenir la défaillance de multiples organes qui tue ces patients. Le contrôle de la voie du NO, ou par une inhibition sélective de la NO synthétase, ou par l'administration de NO exogène, est une des façons de moduler la réponse inflammatoire.

\section{References}

I Gillart T, Bazin JE, Cosserant B, et al. Combined nitric oxide inhalation, prone positioning and almitrine infusion improve oxygenation in severe ARDS. Can J Anaesth 1998; 45: 402-9.

2 Troncy E, Collet J-P, Shapiro $S$, et al. Should we treat acute respiratory distress syndrome with inhaled nitric oxide? (Letter) Lancet 1997; 350: 111-2.

3 Ichinose F, Adrie C, Hurford WE, Bloch KD, Zapol $W M$. Selective pulmonary vasodilation induced by aerosolized zaprinast. Anesthesiology 1998; 88: 410-6.

4 Doering EB, Hanson CW III, Reily DJ, Marshall C, Marsball $B E$. Improvement in oxygenation by phenylephrine and nitric oxide in patients with adult respiratory distress syndrome. Anesthesiology 1997; 87: 18-25.

5 Jolliet P, Bulpa P, Ritz M, Ricou B, Lopez J, Chevrolet J-C. Additive beneficial effects of the prone position, nitric oxide, and almitrine bismesylate on gas exchange and oxygen transport in acute respiratory distress syndrome. Crit Care Med 1997; 25: 786-94.

6 Amato MBP, Barbas CSV, Medeiros DM, et al. Effect of a protective-ventilation strategy on mortality in the acute respiratory distress syndrome. $\mathrm{N}$ Engl J Med 1998; 338: 347-54.

7 Weg JG, Anzueto A, Balk RA, et al. The relation of pneumothorax and other air leaks to mortality in the acute respiratory distress syndrome. $\mathrm{N}$ Engl J Med 1998; 338: 341-6.

8 Stewart TE, Meade MO, Cook DJ, et al. Evaluation of a ventilation strategy to prevent barotrauma in patients at high risk for acute respiratory distress syndrome. N Engl J Med 1998; 338: 355-61.

9 Petros AJ, Marshall JC, pan Saene HK. Should morbidity replace mortality as an endpoint for clinical trials in intensive care? Lancet 1995; 345: 369-71.

10 Guinard N, Beloucif S, Gatecel C, Mateo J, Payen D. Interest of a therapeutic optimization strategy in severe ARDS. Chest 1997; 111: 1000-7. 